\title{
Extended Spectrum Beta Lactamases Detection Using Two Phenotypic Methods Among Uropathogenic Escherichia Coli.
}

\author{
Chauhan S, Khanduri A, Mahawal B S. \\ Department of Microbiology, Shri Guru Ram Rai Institute of Medical and Health Sciences, Patel Nagar, \\ Dehradun.India.
}

\begin{abstract}
Background:Extended spectrum $\beta$-lactamase $(E S B L)$ is an important mechanism of beta-lactam resistance among Gram negative bacteria. The ESBL detection method as described by Clinical Laboratory Standards Institute(CLSI) is used routinely. This method however is not able to detect ESBL in presence of AmpC which is another important mechanism of drug resistance among these bacteria. This study was thus undertaken to compare CLSI recommended method with Cefepime-clavulanic acid method.

Materials and Methods: A total of 216 non repetitive (Escherichia coli) E.coli isolates from urine samples were screened for resistance to Cefpodoxime and Cefotaxime.Those with screening test positive isolates were confirmed for ESBL production using the two phenotypic detection methods.

Results:Among total 216E.coli isolates only 128(59\%) were potential ESBL producers. Of these 109 were positive by using CLSI recommended method while 112 by using Cefepime-clavulanic acid method.Conclusion:Use of Cefepime-clavulanic acid increases sensitivity for ESBL detection in presence of AmpC.
\end{abstract}

Keywords: AmpC $\beta$-lactamase,Cefepime,Ceftazidime,Extended Spectrum $\beta$-lactamase

\section{Introduction}

Extended spectrum $\beta$-lactamase (ESBL) are $\beta$-lactamases capable of conferring bacterial resistance to the Penicillins, first, second, and third- generation cephalosporins, and Aztreonam (but not the Cephamycins or Carbapenems) by hydrolysis of these antibiotics, and which are inhibited by $\beta$-lactamase inhibitors such as clavulanic acid.$^{[1]}$ ESBLs belong to group 2 be of Bush's functional classification. AmpC $\beta$-lactamases belong to group I of Bush's functional classification and are susceptible to advanced spectrum cephalosporins(i.e. cefepime and cefpirome). ${ }^{[2]}$

The ESBL confirmation method has been established by CLSI and is used worldwide. ${ }^{[3]}$ This test however fails to detect ESBL in the presence of AmpC $\beta$ lactamases (AmpC). ${ }^{[4]}$ Several methods have been described for detection of ESBL in presence of AmpC but these may not be routinely used.

The aim of the present study is to find the prevalence of ESBL producing $E$. coli causing urinary tract infections and to compare the two ESBL detection methods one given by CLSI using Ceftazidime-clavulanic acid and the other Cefepime-clavulanic acid .

\section{Materials and Methods:}

A total of 216 non repetitive E.coli isolates obtained from cultures of urine received between January 2012 to August 2012 were included in the study. Identification of these $E$. coli isolates was done using standard microbiological techniques. Antibiotic susceptibility testing was done according to CLSI recommended Kirby Bauer disk diffusion method ${ }^{[3]}$

Each of these E.coli isolates were screened for possible ESBL production by using $30 \mu \mathrm{g}$ ofCefotaxime and $10 \mu \mathrm{g}$ of Cefpodoxime disk.

Every isolate that showed resistance to these screening agents was tested for ESBL detection by using

(i)CLSI recommended method and (ii )cefepime-clavulanic acid method.

The interpretation of results was as follows:

1.A $\geq 5 \mathrm{~mm}$ increase in the zone diameter of the Ceftazidimealone and in combination with Clavulanicacid was indicative of ESBL production as per CLSI.

2. A $\geq 5 \mathrm{~mm}$ increase in the zone diameter of the Cefepime alone and in combination with Clavulanic acid was indicative of ESBL production even in presence of AmpC ${ }^{[5,6,7]}$

E.coliATCC 25922 was taken as negative control and K. pneumoniaeATCC 700603 served as positive control.

\section{Results and Discussion:}

A total of 1136 urine samples were received in the laboratory during the study period out of which $216 \mathrm{E}$.coli were isolated.These 216E.coli isolates were then screened for ESBL production by CLSI recommended screening method using $30 \mu \mathrm{g}$ of Cefotaxime and $10 \mu \mathrm{g}$ of Cefpodoxime.Though, any third generation 
Extended Spectrum Beta Lactamases detection using two phenotypic methods among Uropathogeni... cephalosporin can be used, some workers have recommended Cefpodoxime disk as a good screening agent for ESBL detection in E. coli. ${ }^{[8]}$

The number of potential ESBL producers identified by CLSI recommended screening test were 128 $(59 \%)$.

All screening test positive isolates were further tested for confirmation of ESBL production by CLSI recommended Phenotypic confirmatory test using Ceftazidime and Ceftazidime - Clavulanic acid and Disk Diffusion Method using Cefepime and Cefepime-Clavulanic acid.

Not all 128 screening test positive isolates were confirmed to be ESBL producers. Similarly other studies have also demonstrated that not all screen positive isolates were ESBL producers. ${ }^{[9,10]}$

Out of 128 screen test positive 109 (85.2\%) isolates were positive by CLSI recommended method,112 (87.5\%) were positive byCefepime-Clavulanic acidmethod. Thus 3 isolates of E.coli would have been missed by using only CLSI recommended method.As shown in Table 1(A) and 1(B).This difference was found to be statistically significant ( $\mathrm{p}$ value $<0.05$, Chi square test, Graph Pad).

The ESBL isolates showed maximum sensitivity to Imipenem and Tigecycline followed by Nitrofurantoin as shown in Figure:1.

In our study ESBL along with AmpC production accounted for $2.3 \%$ of E.coli isolates which were detected using Cefepime. Similar study from Chandigarh reported $2 \%$ of isolates having co-existence of Amp C and ESBLs. ${ }^{[11]}$ A study from Haldwani reported as high as $25 \%$ of ESBL and Amp C co-production. ${ }^{[12]}$

In conclusion, the results of the study indicate that the current CLSI recommended method to confirm ESBL enzymes by conducting clavulanate synergy tests with Ceftazidime may be insufficient for ESBL detection in clinical isolates of E.coli since this organism may produce multiple $\beta$-lactamases. In such situations, where AmpC $\beta$-lactamase can interfere with clavulanate synergy, Cefepime clavulanate could be a more sensitive alternative for the detection of ESBL producing organisms. This is consistent with other studies. ${ }^{[13]}$

\section{Acknowledgement}

We are thankful to Mr. Durgesh Ramola, Senior Lab. Technician for his help in carrying out this study.

\section{References}

[1]. Bradford PA.Extended-spectrum beta-lactamases in the $21^{\text {st }}$ century:Characterization,epidemiology,and detection of this important resistance threat.ClinMicrobiol Rev 2001; 14:933-5.

[2]. Bush K, Jacoby G, Medeiros A. A functional classification scheme for beta-lactamases and its correlation with molecular structure. Antimicrob Agents Chemother. 1995;39:1211-33.

[3]. Clinical and Laboratory Standards Institute. Performance standard for antimicrobial susceptibility testing. 17th informational supplement 2007;27(1):M100-S17.

[4]. Paterson DL,Bonomo RA. Extended-spectrum -lactamases :a clinical update.Clin Microbiol Rev 2005;18:657-86.

[5]. Shoorashetty RM, Nagarathnamma T, Prathibha J. Comparison of the boronic acid disk potentiation test and cefepime-clavulanic acid method for the detection of ESBL among AmpC-producing Enterobacteriaceae. Indian J Med Microbiol. 2011;29:297-301.

[6]. Garrec H.Comparsion of Nine Phenotypic Methods for Detection of Extended-Spectrum $\beta$-lactamase Production by Enterobacteriaceae.J Clinic Microbiol.2011;49:1048-1057.

[7]. Derbyshire H, Kay G, Evans K, Vaughan C, Kavuri U, Winstanley T. A simple disc diffusion method for detecting AmpC and extended-spectrum beta-lactamases in clinical isolates of Enterobacteriaceae. J Antimicrob Chemother. 2009 Mar;63:497-501.

[8]. Thomson KS, Sanders CC. A simple and reliable method to screen isolates of Escherichia coli and Klebsiella pneumoniae for the production of TEM- and SHV-derived extended-spectrum $\beta$-lactamases. Clinical Microbiology and Infection. 1997;3:549-54.

[9]. Sridhar Rao P, Basavaraj Khanduri A, Thakuria B, Gupta P. Effectiveness Of Combination Drugs In Extended Spectrum Beta Lactamases Producing Gram Negative Isolates- Experience In A Tertiary Care Hospital Of Uttarakhand. Int J Pharm Bio Sci. 2012;3:251-60.

[10]. Khanduri A, Thakuria B, Gupta P. Effectiveness Of Combination Drugs In Extended Spectrum Beta Lactamases Producing Gram Negative Isolates- Experience In A Tertiary Care Hospital Of Uttarakhand. Int J Pharm Bio Sci. 2012;3:251-60.

[11]. Gupta V. Detection of ESBLs using third and fourth generation cephalosporins in double disc synergy test. Indian J Med Res. 2007;126:486-7.

[12]. Rawat V, Singhai M, Verma PK. Detection of Different beta-Lactamases and their Co-existence by Using Various Discs Combination Methods in Clinical Isolates of Enterobacteriaceae and Pseudomonas spp. J Lab Physicians. 2013;5:21-25.

[13]. Mohanty S, Singhal R, Sood S, Dhawan B, Das B, Kapil A. Comparative in vitro activity of beta-lactam/beta-lactamase inhibitor combinations against gram negative bacteria. Indian J Med Res. 2005;122:425-8.

Table 1(A) Comparison of CLSI Phenotypic confirmatory test using Ceftazidime/Ceftazidime-Clav with Cefepime/Cefepime-Clav:

\begin{tabular}{|l|l|l|l|l|c|}
\hline $\begin{array}{l}\text { Study } \\
\text { Isolate }\end{array}$ & \multirow{2}{*}{$\begin{array}{l}\text { Total } \\
\text { number }\end{array}$} & \multicolumn{2}{|l|}{ Positive by Ceftazidime/Ceftazidime-Clav } & \multicolumn{2}{l|}{ Positive by Cefepime/Cefepime-Clav } \\
\cline { 3 - 6 } & & Number & $\%$ & Number & 87.5 \\
\hline E.coli & 128 & 109 & 85.2 & 112 & \\
\hline
\end{tabular}


Extended Spectrum Beta Lactamases detection using two phenotypic methods among Uropathogeni...

Table 1(B): Comparision of CLSI Phenotypic confirmatory test using Ceftazidime/Ceftazidime- Clav with Cefepime/ Cefepime- Clav:

\begin{tabular}{|c|c|c|c|c|}
\hline $\begin{array}{c}\text { Study } \\
\text { Isolate }\end{array}$ & $\begin{array}{c}\text { Only Positive by Ceftazidime/ } \\
\text { Ceftazidime-Clav }\end{array}$ & $\begin{array}{c}\text { Only Positive by } \\
\text { Cefepime/Cefepime-Clav }\end{array}$ & $\begin{array}{c}\text { Positive by } \\
\text { Both }\end{array}$ & $\begin{array}{c}\text { Negative by } \\
\text { Both }\end{array}$ \\
\hline E.coli & 00 & 03 & 109 & 16 \\
\hline
\end{tabular}

Figure1:Sensitivity pattern of ESBL isolates.

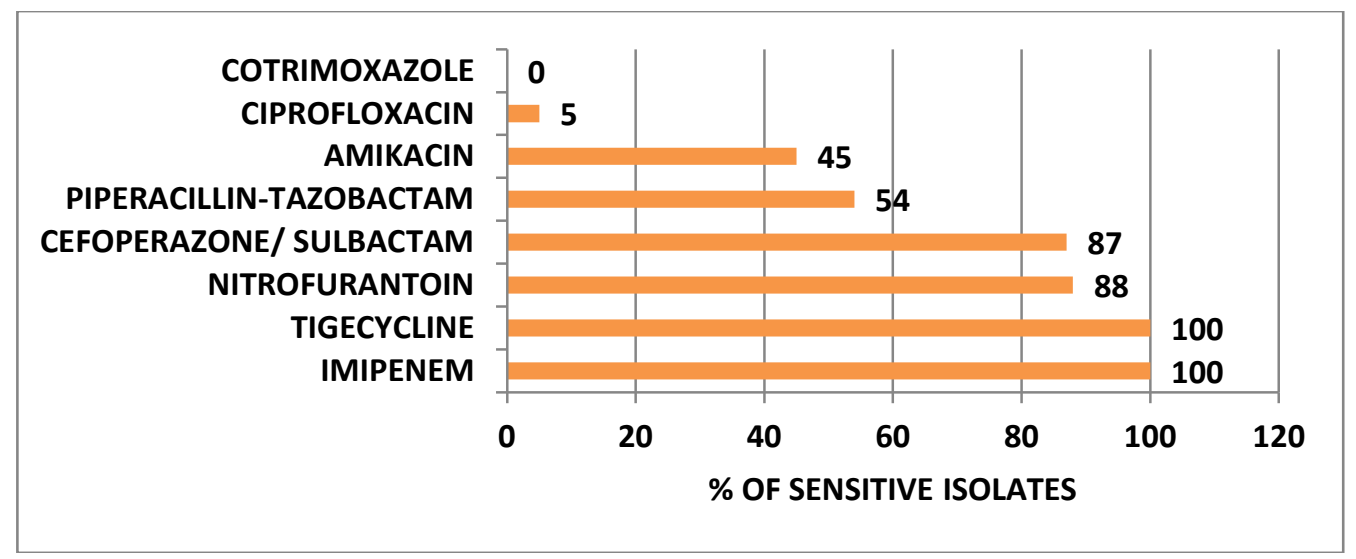

